\title{
Aplicação do modelo Weibull na modelação matemática para análise da viabilidade de geração de energia eólica
}

\author{
Gracielle Antunes Araújo ${ }^{1}$ \\ Diana Darlen Soares Cangussú ${ }^{2}$ \\ Míriam Ramos Silva ${ }^{3}$ \\ Karoline Martins Perdigão ${ }^{4}$
}

\begin{abstract}
RESUMO
O aumento do consumo de energia elétrica resultou nos últimos anos em racionamentos e apagões, levando à ampliação da geração centralizada para produção de energia. As microgerações de energia com fontes renováveis vem crescendo nos últimos anos, sendo, uma destas, a eólica, cujo potencial depende do clima local, já que exige um ambiente adequado para a implantação. Assim, esta pesquisa é motivada pela necessidade de obter uma ferramenta robusta para controle e aplicação da geração de energia elétrica a partir do potencial eólico em ambientes favoráveis. Para o estudo foi considerado o levantamento de dados a partir de um anemômetro instalado na laje do Bloco B do Centro Universitário Doctum de Teófilo Otoni, para mensurar a velocidade incidente do vento no local. Aplicou-se uma análise estatística e investigou-se o ajustamento a um modelo matemático de valores extremais para máximos. Concluiu-se que os dados seguem uma distribuição de probabilidade Weibull de máximos, com parâmetros estimados pelo método da máxima verossimilhança, sendo este modelo uma ferramenta que servirá como suporte para aplicação de projetos de instalação de energia eólica.
\end{abstract}

Palavras-chave: Distribuição de Weibull. Microgeração Distribuída. Microgeração Eólica.

\footnotetext{
${ }^{1}$ Graduada em Matemática Aplicada e Mestra em Estatística, ambos pela Faculdade de Ciências da Universidade de Lisboa - FCUL, Portugal. Doutoranda em Estatística pela Universidade Federal de Minas Gerais - UFMG. É professora e pesquisadora da Universidade do Estado de Minas Gerais - UEMG, Unidade de João Monlevade, MG, Brasil. E-mail: gracielle.araujo@uemg.br.

${ }^{2}$ Graduada em Engenharia Civil pela Universidade Vale do Rio Doce - UNIVALE. Possui Especialização em Estruturas pela Escola de Engenharia da Universidade Federal de Minas Gerais - UFMG e mestrado em Engenharia Civil pela Universidade Federal do Ceará - UFC. É professora e pesquisadora da Universidade do Estado de Minas Gerais - UEMG, Unidade de João Monlevade, MG, Brasil. E-mail: darlencangussu@gmail.com.

${ }^{3}$ Engenheira Elétrica formada pela Faculdades Unificadas de Teófilo Otoni - FUTO. Cursa Especialização em Gerenciamento de Projetos pela PUC Minas e Especialização em Engenharia Elétrica pela Faculdade EducaMais - UNIMAIS, MG, Brasil. E-mail: smiriam2005@gmail.com.

${ }^{4}$ Graduanda em Engenharia Civil pela Universidade do Estado de Minas Gerais - UEMG, Unidade de João Monlevade, e estagiária em Engenharia Civil na empresa L\&L Engenharia S/A (Construtora Limac), MG, Brasil. E-mail: kmartinsperdigao@gmail.com.
} 


\title{
D Application of the Weibull model in mathematical modeling to analyze the viability of wind energy generation
}

\begin{abstract}
The increase in electricity consumption has resulted in the last few years in rationing and blackouts, leading to the expansion of centralized generation for energy production. Microgenerations of energy with renewable sources have been growing in recent years, one of which, wind power, whose potential depends on the local climate, in which it requires an adequate environment for implementation. Thus, this research is motivated by the need to obtain a robust tool for the control and application of electricity generation from wind potential in favorable environments. For the study it was considered the data survey using an anemometer installed on the slab of Block B of the Doctum University Center of Teofilo Otoni, to measure the incident wind speed in the place. A statistical analysis was applied and the adjustment to a mathematical model from extreme to maximum values was investigated. It was concluded that the data follow a Weibull probability distribution of maxima, with parameters estimated by the maximum likelihood method, this model being a tool that will serve as a support for the application of wind energy installation projects.
\end{abstract}

Keywords: Weibull Distribution. Distributed Microgeneration. Wind Microgeneration.

Artigo recebido em: 06/05/2020

Aceito em: 19/06/2020 


\section{INTRODUÇÃO}

Devido ao aumento da população e do surgimento de novas tecnologias, o consumo de energia elétrica aumentou, mas a oferta não se expandiu na mesma proporção, fazendo com que houvesse racionamentos e apagões. Como consequência, foi necessária a ampliação da geração centralizada em grandes centrais hidrelétricas e termelétricas, apesar do atraso devido aos altos custos.

A geração distribuída vem crescendo muito nos últimos anos e tem como atrativo a utilização de fontes renováveis, sendo consideradas como uma alternativa para as concessionárias e como uma vantagem para o consumidor, o que faz com que a geração de energia seja produzida e consumida no local de instalação. Caracteriza-se em centrais geradoras de energia elétrica, de qualquer potência, com instalações conectadas diretamente no sistema elétrico de distribuição ou através de instalações de consumidores (CEMIG, 2019). Por esse motivo, esse tipo é considerado como geração distribuída, por não depender de uma central elétrica para gerar e entregar a energia, sendo seu próprio ponto de entrega e de consumo.

Recursos energéticos distribuídos são definidos como tecnologias de geração, armazenamento de energia elétrica e redução do consumo localizados dentro dos limites da área de uma determinada concessionária de distribuição, normalmente junto a unidades consumidoras, atrás do medidor behind-the-meter (BRASIL, 2020). Nesse sentido, um dos recursos energéticos distribuídos considerados atualmente no Brasil é a microgeração distribuída, central geradora de energia elétrica, com potência instalada menor ou igual a 75 kW e que utiliza cogeração qualificada ou fontes renováveis de energia elétrica conectada na rede de distribuição por meio de instalações de unidades consumidoras (ANEEL, 2015; CEMIG, 2019).

A microgeração distribuída com fonte eólica - microgeração eólica - utiliza-se da velocidade de dispersão dos movimentos do ar na atmosfera terrestre (ventos) para geração de energia eólica próxima ao consumidor, proporcionando redução de perdas de tensão dos cabos de distribuição e da tarifa de energia elétrica. Utiliza-se do Sistema de Compensação de Energia Elétrica, no qual a energia ativa injetada por unidade consumidora com microgeração distribuída é cedida, por meio de empréstimo gratuito, à distribuidora local e posteriormente compensada com o consumo de energia elétrica ativa (ANEEL, 2015). 
A microgeração eólica necessita do vento para seu funcionamento, o que se torna uma limitação para a instalação desse sistema. Existe a necessidade de uma velocidade mínima de $2 \mathrm{~m} / \mathrm{s}$ a 10 metros de altura para que o microaerogerador entre em funcionamento e a energia produzida seja viável para o local, considerando que alguns locais não atingem essa velocidade.

A energia eólica é uma importante fonte renovável, abundante, limpa, mostrando-se extremamente competitiva frente às demais tecnologias candidatas à expansão (BRASIL, 2020). Entretanto, essa fonte de energia é intermitente, dependendo das condições atmosféricas, com alteração de região para região. É necessário um monitoramento da velocidade do vento, em que se verifica a viabilidade de instalação de aerogeradores e ainda um ambiente amplo, sem muita interferência de edificações e de árvores. Tudo isso torna mais difícil a elaboração de previsões sobre a quantidade de energia a ser gerada e, portanto, menos competitiva em relação às outras formas de geração, podendo atender à demanda com uma menor incerteza.

Como consequência, esse sistema de microgeração eólica pode tanto ser um bom investimento quanto não, visto que o vento não é constante, sendo necessário o dimensionamento correto das turbinas para que possam gerar de acordo com a localidade, o que faz com que a energia gerada seja um complemento da unidade consumidora. Mesmo sendo um complemento, ela também poderá contribuir na compressão de emissão de gás carbônico $\left(\mathrm{CO}_{2}\right)$, porque o vento é um meio natural e sustentável, não lançando gases poluentes na atmosfera.

Assim, esta pesquisa é motivada pela necessidade de agregar mais uma ferramenta, considerando-a robusta para controle e aplicação da geração de energia a partir do potencial eólico em ambientes favoráveis. Para isso aplicou-se o ajustamento dos dados, levantados a partir de um anemômetro instalado na laje do Bloco B do Centro Universitário Doctum de Teófilo Otoni (UniDoctum), para mensurar a velocidade incidente do vento no local.

Chegou-se à conclusão de que a distribuição de probabilidade Weibull de máximos se ajusta aos dados, com parâmetros estimados pelo método da máxima verossimilhança, sendo este modelo uma ferramenta de controle o qual pode servir como suporte para a aplicação de projetos de instalação de energia eólica. 


\section{IMPORTÂNCIA DA MICROGERAÇÃO DE ENERGIA EÓLICA}

Atualmente, a microgeração de energia no Brasil vem se tornando fundamental devido à sobrecarga que existe no sistema elétrico, sendo necessário grandes usinas hidrelétricas para que seja viável sua distribuição para todas as unidades consumidoras. A microgeração faz com que essas unidades consumidoras possam gerar e consumir a sua própria energia, fazendo com que diminua a do sistema elétrico brasileiro em relação à distribuição de energia quando instalado esse tipo de geração em grande demanda.

Devido ao aumento da demanda, houve crescimento da geração centralizada em grandes centrais hidrelétricas e termelétricas, com participação na capacidade instalada de geração elétrica no Brasil, em MW, de $60.4 \%$ e $24.9 \%$, respectivamente, em 2018 (BRASIL, 2019).

A microgeração de energia com fontes renováveis, que caracteriza-se como uma preocupação urgente considerando-se que os processos convencionais de geração de energia elétrica estão entre os principais responsáveis pelas emissões de gases de efeito estufa (PAZHERI; OTHMAN; MALIK, 2014), apresenta-se como uma das melhores propostas para solução de problemas ambientais e diminuição de tarifas de energia.

Observa-se na Figura 1 que o Brasil dispõe de uma matriz de energia elétrica de origem predominantemente renovável, com destaque para a fonte hidráulica que responde por 64.7 $\%$ da oferta interna brasileira.

Figura 1: Geração de energia elétrica por fonte no Brasil

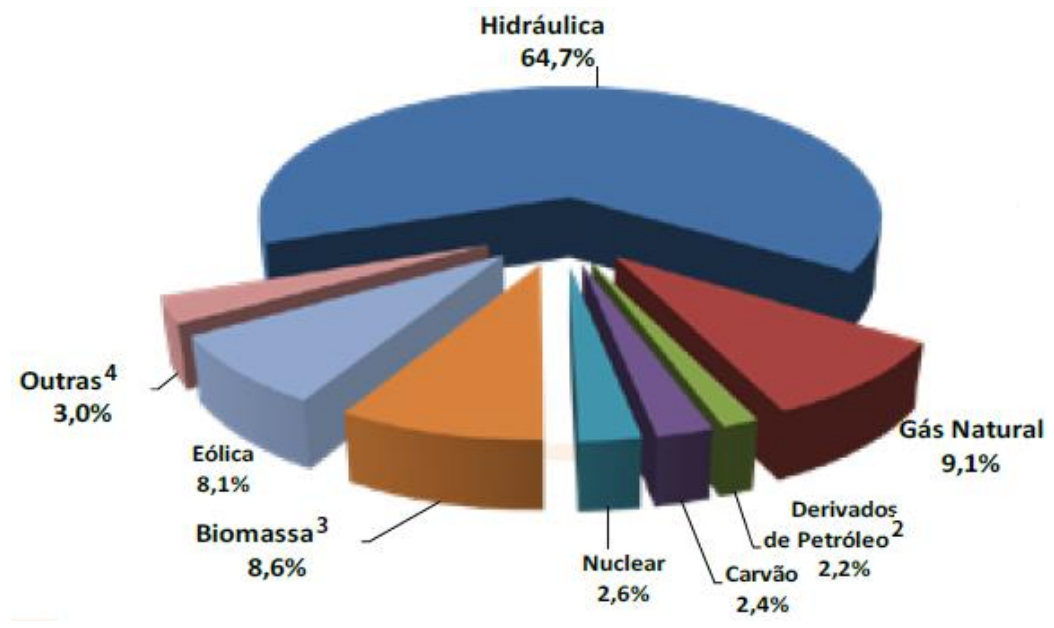

Fonte: Brasil (2019). 
O mundo tem $4 \%$ de energia eólica (BRASIL, 2019) e considera-se que, nos últimos seis anos, a evolução da produção de energia eólica no Brasil foi altamente positiva. A Figura 1 mostra a geração de energia, em GWh, em que a fonte eólica corresponde a $8.1 \%$ da oferta brasileira (BRASIL, 2019) e se destaca como uma alternativa em relação às grandes centrais de geração, dependendo somente do clima do local para a geração. Frente à política de expansão energética brasileira e à crescente inserção da fonte renovável eólica, entende-se que o sistema está passando por um processo de adaptação (JONG; KIPERSTOK; TORRES, 2015; SCHMIDT; CANCELLA; PEREIRA JÚNIOR, 2016).

Assim, a geração distribuída com fonte eólica ainda vem se desenvolvendo no país, com somente 40 conexões (Figura 2) mesmo o Brasil tendo locais com climas ideais para a instalação de microgeradores e ventos proporcionais para a geração noturna.

Figura 2: Número de conexões por fonte no Brasil

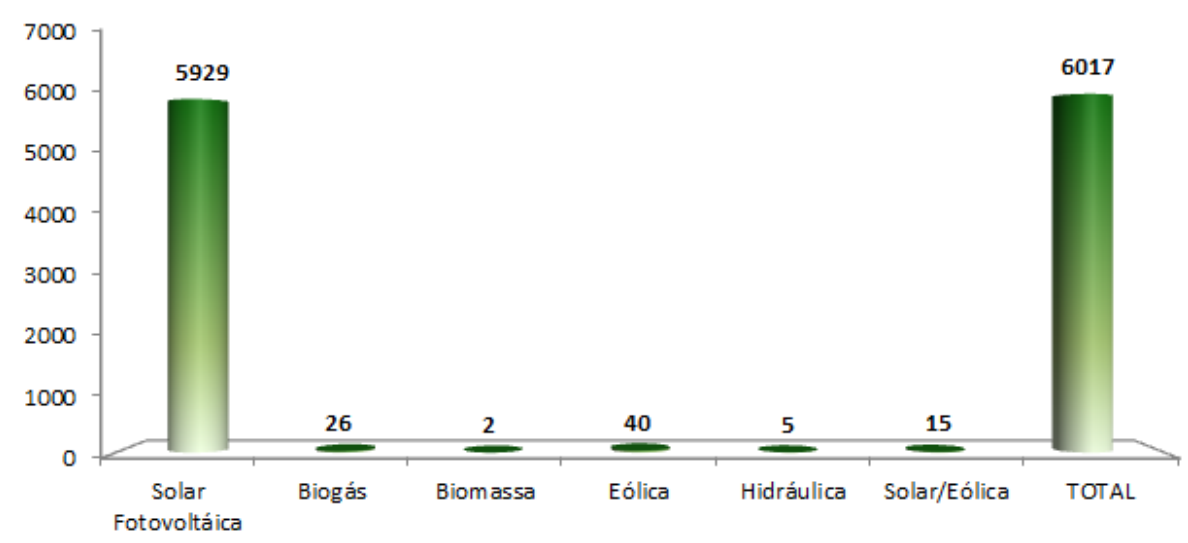

Fonte: Brasil (2019).

O número de conexões da fonte eólica totaliza uma potência de 159 kW (Figura 3).

Figura 3: Potência instalada

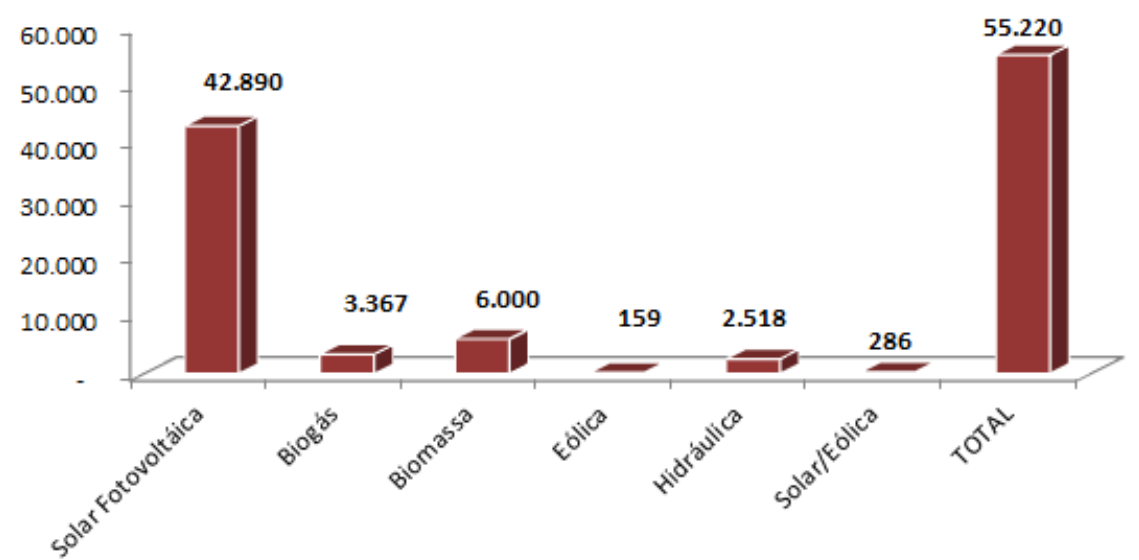

Fonte: Brasil (2019). 
Esse valor é baixo quando comparado ao da microgeração de energia solar, mas é de grande importância visto que as centrais geradoras de energia elétrica deixaram de enviar essa quantidade até seus pontos de entrega, especialmente em locais onde a rede tem uma menor capacidade de recepção.

A capacidade instalada de geração de energia elétrica no Brasil a partir da fonte eólica alcançou 14390 MW em 2018, equivalente a um aumento de $17.2 \%$ em relação ao ano anterior, quando se atingiu $12283 \mathrm{MW}$ (BRASIL, 2019).

A sustentabilidade tem assumido papel importante nas empresas de geração de energia elétrica, estando relacionada ao equilíbrio entre as dimensões social, econômica e ambiental. Também se mostra importante na microgeração distribuída com fonte eólica, pois, quando é gerada por fontes renováveis, deixa de emitir poluentes causadores do efeito estufa, beneficiando o ambiente e a população não somente com a não emissão de gases, mas também com a geração de empregos e crescimento econômico.

O Brasil, porém, ainda tem um longo caminho para melhorar a competitividade e garantir a sustentabilidade de longo prazo da fonte eólica de energia (MELO, 2014).

\section{MÉTODO DE PESQUISA}

\subsection{Caracterização do local de instalação do anemômetro}

A viabilidade da geração de energia eólica depende da incidência do vento e, para ser aplicada, a importância resulta da necessidade da caracterização do vento para a elaboração de projetos eólicos. Com isso, faz-se necessário um estudo do local.

O local para a instalação do anemômetro estava na área apresentada na Figura 4, no Centro Universitário Doctum de Teófilo Otoni (UniDoctum), na cidade de Teófilo Otoni, estado de Minas Gerais.

Os parâmetros incluídos na escolha do local foram:

a) dados do vento local: quanto às características de vento da região;

b) condições topográficas viáveis: levantamento da área por meio de análise de imagens e posterior visita técnica in loco; 
c) condição da cobertura da edificação: análise para determinar o tipo de suporte utilizado para instalação do anemômetro;

d) fácil acesso à rede elétrica;

e) análise de viabilidade técnica de implantação de tecnologias ambientais no UniDoctum.

Figura 4: Localização do UniDoctum

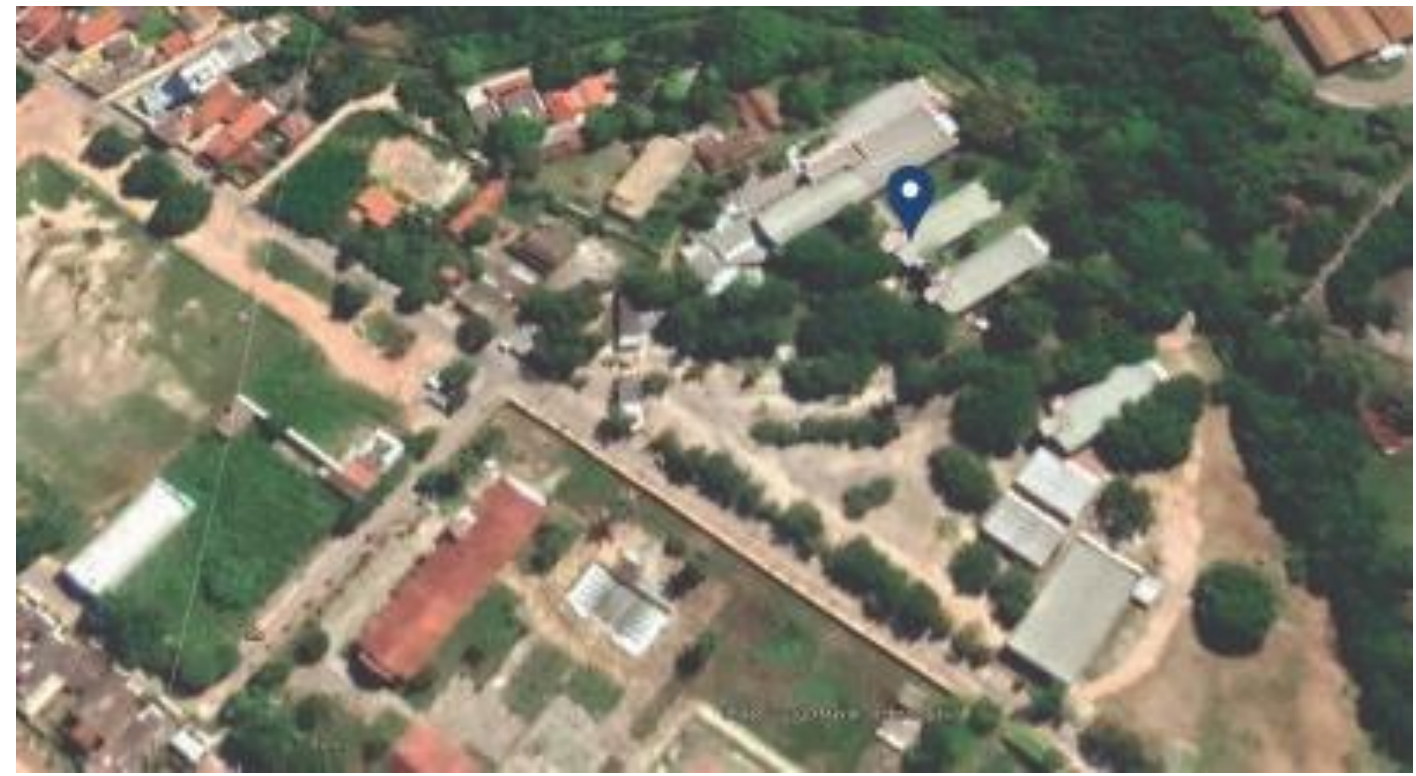

Fonte: Google Maps (2016).

\subsection{Instalação do anemômetro}

O anemômetro é um instrumento meteorológico com a função de medir a velocidade instantânea do vento local e pode apresentar diferentes níveis de precisão a depender da aplicação desejada. É por isso amplamente utilizado na identificação de seu histograma em regiões onde se pretende desenvolver projetos eólicos (BRAGA, 2013) e, principalmente, em estações meteorológicas para conhecer o deslocamento de massas de ar do local.

Instalou-se um anemômetro na laje do segundo pavimento do Bloco B do UniDoctum, ilustrado na Figura 5-A, com altura de medição de $10 \mathrm{~m}$, considerando $3.15 \mathrm{~m}$ de pé direito de cada um dos 3 andares do Bloco $\mathrm{B}$, acrescida de $0.55 \mathrm{~m}$ relativa ao mastro de suporte do anemômetro, conforme a Figura 5-B, com o objetivo de mensurar a velocidade incidente do vento no local para determinação do potencial eólico da área escolhida, em um período de 
duas quinzenas intercaladas, em dias aleatórios, a partir da aplicação do método de amostragem aleatória simples.

Figura 5: Anemômetro instalado na laje

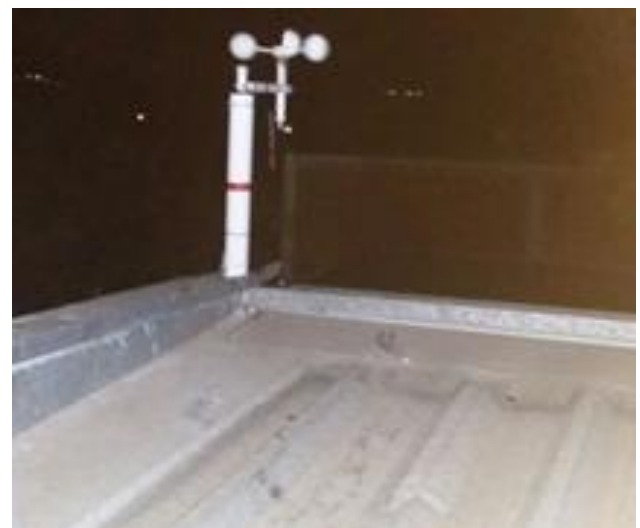

(A)

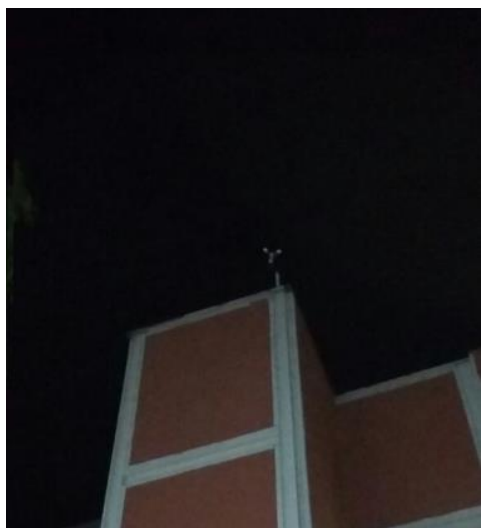

(B)

Fonte: Autoria própria.

O anemômetro de concha usado (Figura 5-A) era composto, conforme a Figura 6, por:

a) mastro em alumínio marítimo, com comprimento de $0.55 \mathrm{~m}$;

b) tubo para base em alumínio marítimo, com Ø 2";

c) três copos “conchas" em alumínio marítimo;

d) indicador de direção do vento em alumínio - visual;

e) leitor digital com calibração de fábrica.

Figura 6: Componentes do anemômetro

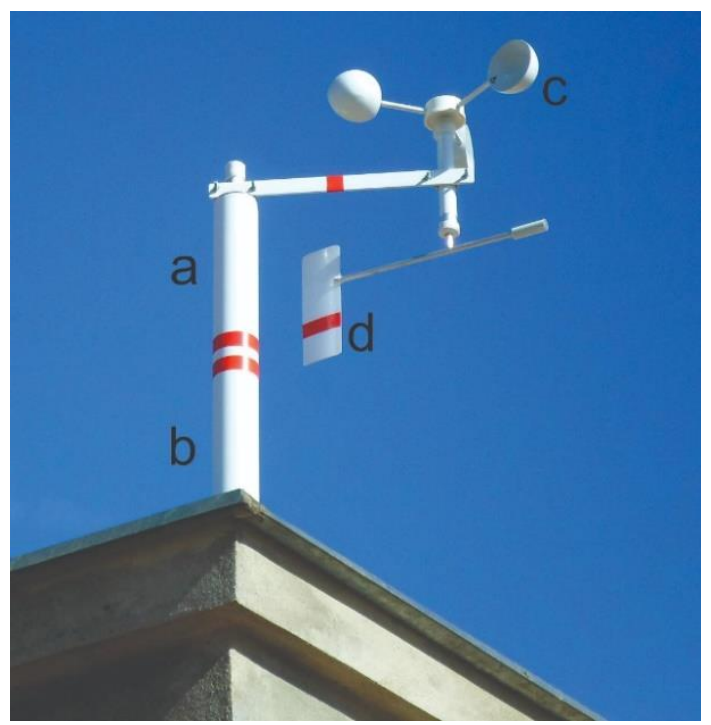

Fonte: Autoria própria. 


\subsection{Levantamento de dados}

De maneira geral, o levantamento de dados para instalação de geração de energia eólica é feito por meio de estações meteorológicas de observação de superfície e mapas eólicos disponíveis nas concessionárias de cada estado, verificando, conforme a Tabela 1, a velocidade média anual do vento e suas possibilidades de uso para energia eólica.

Tabela 1: Velocidade do vento para uso em energia eólica

\begin{tabular}{|c|c|}
\hline $\begin{array}{l}\text { Velocidade média anual a } 10 \text { metros } \\
\text { de altura acima do } 50 l o(\mathrm{~m} / \mathrm{s})\end{array}$ & Possibilidade de uso para energia eólica \\
\hline Abaixo de 3 & Usualmente não viável \\
\hline De 3 a 4 & Opção para bombas eólicas, mas não para geradores \\
\hline De 4 a 5 & Viável para geradores eólicos isolados \\
\hline De 5 a 7 & Viável para bombas eólicas e geradores eólicos isolados \\
\hline Acima de 7 & $\begin{array}{l}\text { Viável para bombas eólicas e geradores eólicos isolados } \\
\text { e conectados à rede }\end{array}$ \\
\hline
\end{tabular}

Fonte: Bohme et al. (2016).

Por meio disso, analisou-se a viabilidade da microgeração eólica e a possibilidade da escolha do aerogerador.

Para mensurar a velocidade do vento, instalou-se no anemômetro o velocímetro ciclocomputador com 14 funções, modelo SunDing SD - 548B, utilizado para medir a velocidade alcançada por uma bicicleta. Configurou-se para que ele pudesse medir a velocidade do eixo do anemômetro, quando este estava em rotação. Após essa configuração, ambos estavam aptos a capturar a velocidade do vento, sendo este modelo muito aplicado para pequenas estações meteorológicas.

Após a instalação do anemômetro, houve a necessidade de adequar um smartphone da marca Samsung e modelo Pocket com o software Spy Camera instalado, de plataforma gratuita e disponível no Google Play, para que fossem armazenados os dados através de fotos capturadas a cada cinco minutos (Figura 7), visto que o leitor digital do anemômetro indicava os dados, mas não os armazenava. 
Figura 7: Leitor digital

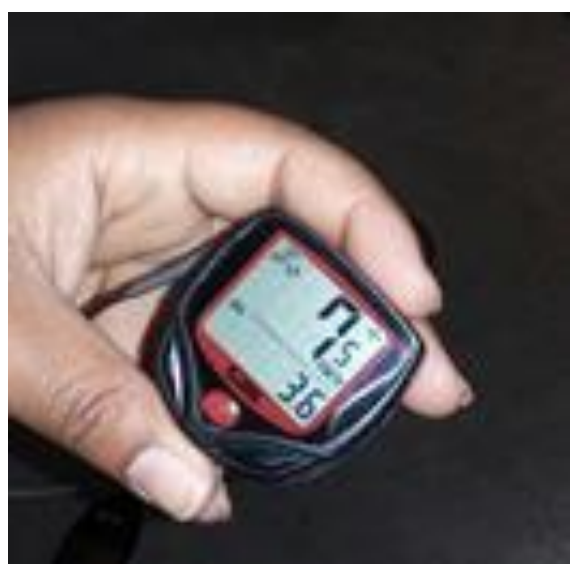

Fonte: Autoria própria.

Por fim, após a adequação, foi feita a verificação diária do funcionamento do software instalado por pausar frequentemente e, quando necessário, ele foi reiniciado com o propósito de gravar todos os dados possíveis.

\subsection{Tratamento dos dados}

Após a execução dos ajustes de todos os empecilhos ocorridos durante o levantamento de dados, estes foram registrados em uma planilha, em que foi montada uma discussão acerca das informações, correlacionando-as e as comparando com dados atuais originários de literaturas técnicas.

Para o tratamento e o estudo dos dados, utilizou-se do software R Project, versão 3.2.4, o qual foi aplicado para conduzir análises estatísticas e dispor os resultados.

\subsection{Ajustamento dos dados}

O ajustamento dos dados foi feito por meio de métodos de distribuição, os quais demonstraram a análise de confiabilidade dos dados coletados. As distribuições de probabilidade são modelos amplamente utilizados para controle e previsão de diversos processos (PESTANA; VELOSA, 2008).

A distribuição de Weibull é muito utilizada na modelação de dados assimétricos e possui um papel muito importante na descrição de processos, sendo bastante aplicada no ramo de análise estatística de dados experimentais, em que esta adaptabilidade é aproveitada 
para empregar o modelo em diversos campos de estudo (ALLAN; BILLINTON, 1992). Seja uma variável aleatória $X \sim \operatorname{Weibull}(\beta, \delta)$, a distribuição é dada por:

$$
f(x ; \beta, \delta)= \begin{cases}\frac{\beta}{\delta^{\beta}} x^{\beta-1} e^{-\left(\frac{x}{\delta}\right)^{\beta},} & x>0 \\ 0 & x \leq 0\end{cases}
$$

Sendo $\beta$ e $\delta$ os parâmetros de escala e forma, respectivamente, no qual a média e a variância são definidas, a partir destes, por:

$$
\begin{aligned}
\mu & =E[X]=\delta \Gamma\left(1+\frac{1}{\beta}\right) \\
\sigma^{2} & =\operatorname{Var}[X]=\delta^{2} \Gamma\left(1+\frac{2}{\beta}\right)-\delta^{2}\left[\Gamma\left(1+\frac{1}{\beta}\right)\right]^{2}
\end{aligned}
$$

A fim de determinar os estimadores do modelo, aplicou-se o Método da Máxima Verossimilhança (MMV). Assim, dada uma amostra de dimensão $n$, seguiu-se os seguintes passos para a aplicação do método, no qual determinou-se:

a) a função verossimilhança dada por:

$$
L\{\beta, \delta\}=\prod_{i=1}^{n} f\left(x_{i} ; \beta, \delta\right)=\prod_{i=1}^{n} \frac{\beta}{\delta^{\beta}} x_{i}^{\beta-1} e^{-\left(\frac{x_{i}}{\delta}\right)^{\beta}}
$$

b) $l\{\beta, \delta\}=\ln (L\{\beta, \delta\})$;

c) resolução do sistema para estimação dos parâmetros:

$$
\left\{\begin{array}{l}
\frac{\partial l\{\beta, \delta\}}{\partial \beta}=0 \\
\frac{\partial l\{\beta, \delta\}}{\partial \delta}=0
\end{array}\right.
$$

d) métodos numéricos para o cálculo do parâmetro de forma.

O MMV determina os estimadores como função do valor do parâmetro que tornariam máxima a verossimilhança da amostra ocorrida (PESTANA; VELOSA, 2008).

\section{RESULTADOS E DISCUSSÕES}




\subsection{Condições da velocidade do vento na região que situa o UniDoctum}

No levantamento de dados, obteve-se valores de velocidade do vento entre 0.00 a 7.29 $\mathrm{m} / \mathrm{s}$, como mostra o Gráfico 1. O local apresentou viabilidade para a instalação de um microaerogerador, mas é necessário que a coleta de dados seja feita em uma altura superior à que foi realizada para garantir melhor aproveitamento da captação, em atendimento ao dado da Tabela 1, o qual mostra que valores acima de $7 \mathrm{~m} / \mathrm{s}$ são viáveis para instalação de bombas eólicas e geradores eólicos isolados e conectados à rede.

Dessa forma, esses equipamentos, quando ligados à rede, a estarão alimentando com energia limpa e renovável. É por esse motivo que velocidades acima de $7 \mathrm{~m} / \mathrm{s}$ são mais eficazes, uma vez que gerariam uma quantidade maior de energia e o aerogerador estaria em trabalho por um maior tempo, evitando que ele fique ocioso.

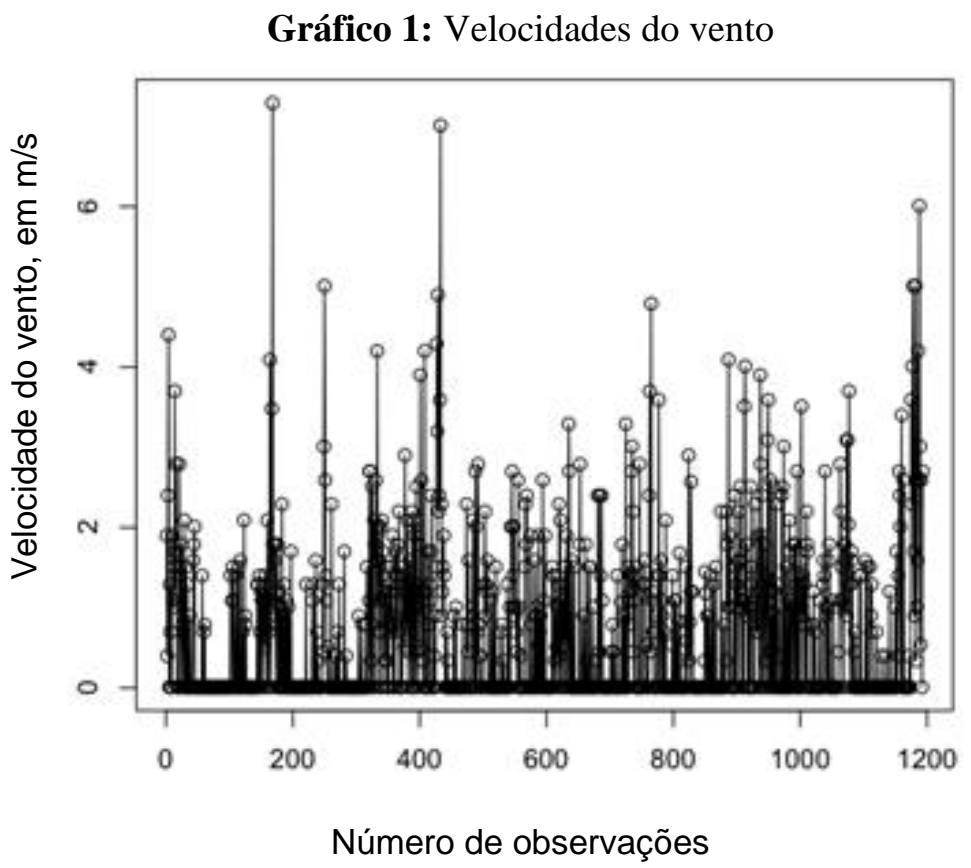

Fonte: R Project (2014).

A Tabela 2 apresenta as principais estatísticas descritivas dos dados. Observa-se que as medidas de localização, média e mediana não estão próximas, o que pode ser justificado pela alta variabilidade, indicando que os dados não possuem uma medida central. Segundo Triola (2008), a comparação entre as medidas de localização, média, moda e mediana pode dizer algo sobre a característica de assimetria dos dados. Observa-se ainda na mesma tabela, que as medidas do coeficiente de assimetria e curtose indicam que os dados não seguem um 
modelo central, sendo que, respectivamente, a primeira indica uma distribuição assimétrica, dando indicações sobre o peso relativo das caudas, e a segunda mede o grau de achatamento de uma distribuição, considerado em relação ao da distribuição normal, sendo assim uma distribuição platicúrtica.

Tabela 2: Estatísticas descritivas dos dados

\begin{tabular}{c|c}
\hline Média & 0.6728835 \\
\hline Mediana & 0 \\
\hline Desvio-padrão & 1.002729 \\
\hline Mínimo & 0.01 \\
\hline Máximo & 7.29 \\
\hline Coeficiente de assimetria & -1.002569 \\
\hline Curtose & 1.006768 \\
\hline
\end{tabular}

Fonte: R Project (2014).

O Gráfico 2 apresenta a concentração dos dados, tendo em consideração os quantis. Verifica-se ainda que os dados têm uma grande variabilidade em torno do ponto central.

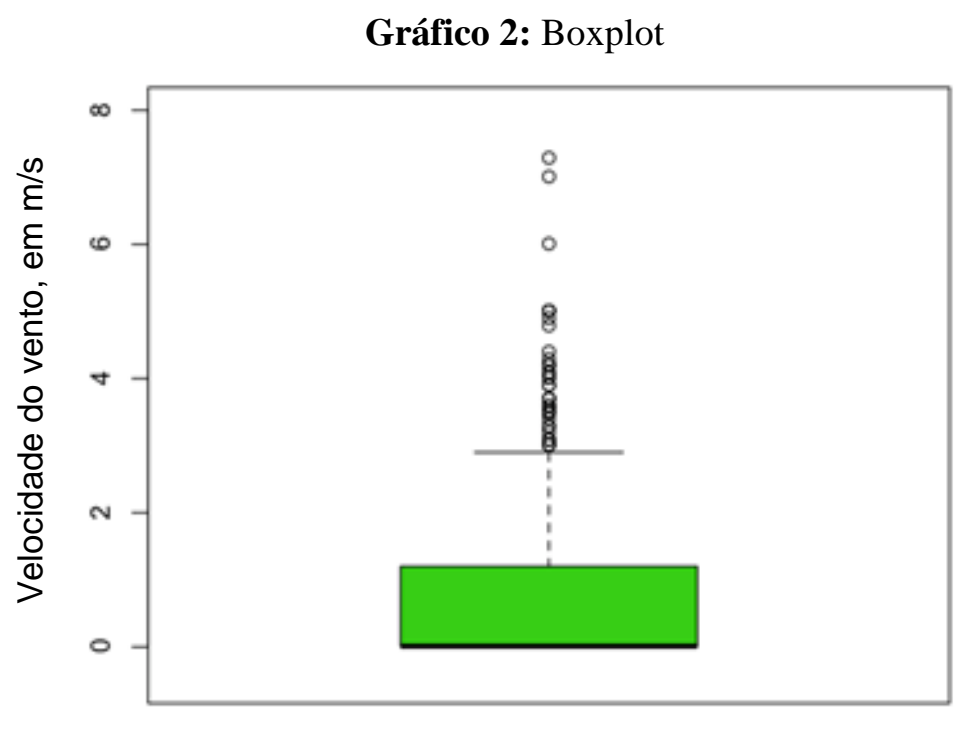

Número de observações

Fonte: R Project (2014). 
A Tabela 3 demonstra as classes dos dados, que são intervalos determinados na construção do histograma (Gráfico 3), em que podemos verificar quantas vezes eles ocorreram, durante o período de análise, em cada classe.

Tabela 3: Classes dos dados

\begin{tabular}{c|c}
\hline $\mathbf{I}_{\mathbf{1}}=[\mathbf{0}, \mathbf{1 . 2 1 3 3 3}[$ & 906 \\
\hline $\mathbf{I}_{\mathbf{2}}=[\mathbf{1 . 2 1 3 3 3 , 2 . 4 2 6 6 6 7}[$ & 211 \\
\hline $\mathbf{I}_{\mathbf{3}}=[\mathbf{2 . 4 2 6 6 6 7 , 3 . 6 4 [}$ & 54 \\
\hline $\mathbf{I}_{\mathbf{4}}=[\mathbf{3 . 6 4}, \mathbf{4 . 8 5 3 3 3 3}[$ & 15 \\
\hline $\mathbf{I}_{\mathbf{5}}=[\mathbf{4 . 8 5 3 3 3 3 , \mathbf { 6 . 0 6 6 6 7 } [}$ & 5 \\
\hline $\mathbf{I}_{\mathbf{6}}=[\mathbf{6 . 0 6 6 6 7}, \mathbf{7 . 2 8}[$ & 2 \\
\hline Total & $\mathbf{1 1 9 3}$ \\
\hline
\end{tabular}

Fonte: R Project (2014).

A partir da análise exploratória e validação informal dos dados, verificou-se que estes podem ser ajustados a um modelo Weibull. Assim, para confirmar essa hipótese, aplicaramse algumas técnicas de ajustamentos no âmbito de confirmar o pressuposto.

A Tabela 4 apresenta os estimadores dos valores dos parâmetros da distribuição de Weibull, aplicando o MMV.

Tabela 4: Estimadores dos valores dos parâmetros da distribuição de Weibull

\begin{tabular}{c|c}
\hline$\widehat{\boldsymbol{\beta}}$ & 0.6404312 \\
\hline$\widehat{\boldsymbol{\delta}}$ & 0.4215533 \\
\hline
\end{tabular}

Fonte: R Project (2014).

No Gráfico 3 é apresentado um histograma, representando uma imagem estatística da função densidade de probabilidade, o que proporciona melhor interpretação e visualização da intensidade dos dados obtidos. Observa-se a função densidade do modelo, com os parâmetros estimados na Tabela 4, em que os valores aproximados de zero são de alta intensidade, fazendo com que o microaerogerador a ser dimensionado seja de pequeno porte e que tenha início de geração de energia eólica próximo a este valor. 


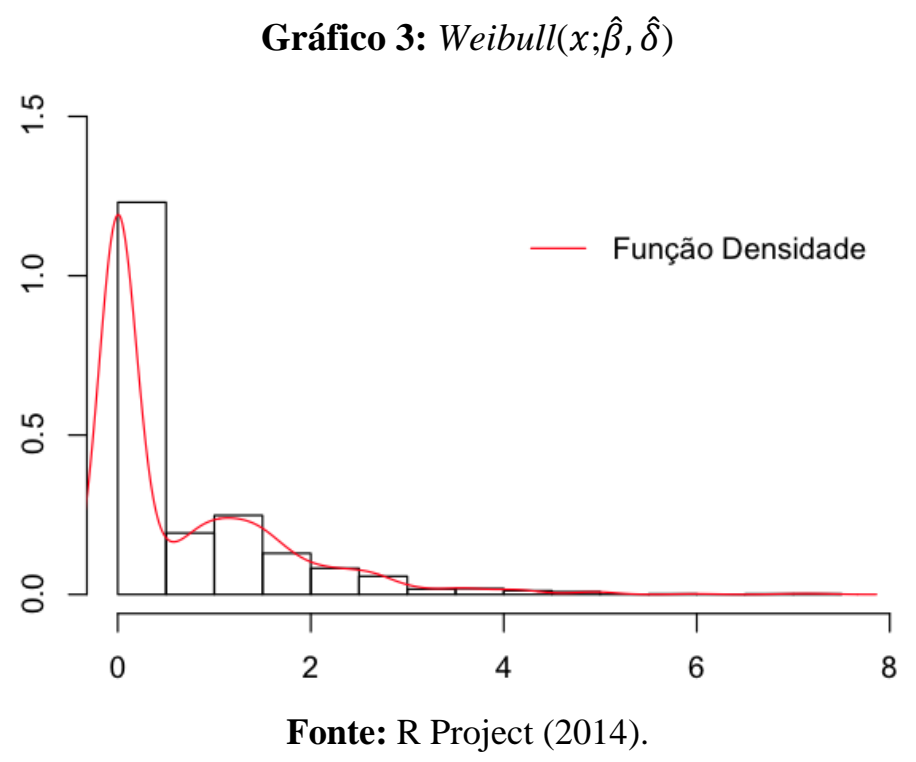

Para avaliar se a amostra segue realmente uma distribuição de Weibull com os parâmetros estimados pelo MMV, aplicou-se o teste do qui-quadradro $\left(\chi_{2}\right)$ e KolmogorovSmirnov, com o qual se pretendeu verificar:

$$
\left\{\begin{array}{l}
\mathrm{H}_{0}: a \text { amostra provém de uma distribuição de Weibull } \\
\mathrm{H}_{1}: a \text { amostra não provém de uma distribuição de Weibull. }
\end{array}\right.
$$

Considerando a comparação entre a estatística teste observada, ET obs, e o valor crítico para a estatística inerente a cada teste, com um nível de significância de $5 \%$, chegou-se às conclusões:

a) para o teste $\chi_{2}$, como $6.417=\mathrm{ET}_{\mathrm{obs}}<7.815$, não rejeitou-se a hipótese nula. Logo, é forte a evidência estatística de que a distribuição é uma Weibull;

b) para o teste de Kolmogorov-Smirnov, como $0.04113=\mathrm{ET}_{\mathrm{obs}}<\frac{1.63}{\sqrt{1193}} 3=$ 0.04719189, não rejeitou-se a hipótese nula. Logo, há razões para acreditar que os dados são modelados pela distribuição de Weibull.

Com o ajustamento desse modelo, objetivou-se mais uma ferramenta robusta de controle e de previsão da curva característica dos ventos incidentes na cidade de Teófilo Otoni - MG, a fim de atuar na consequente tomada de decisão na área de estudo desta pesquisa.

\subsection{Comparação dos dados}

Verificaram-se diferentes velocidades dos ventos durante o levantamento de dados. 
A partir deles, pode-se justificar porque as velocidades dos ventos foram semelhantes às das estações Meteorológicas de Observação de Superfície existentes em Teófilo Otoni - MG.

As Figuras 8 e 9 e a Tabela 5 demonstram essa semelhança, em que a velocidade do vento colhida na Estação Meteorológica de Observação de Superfície Automática, instalada no Aeroporto Kemil Kumaira, situada a uma altura superior à do anemômetro instalado no UniDoctum, capturou uma velocidade de $5.1 \mathrm{~m} / \mathrm{s}$ no dia 30 de setembro de 2016 às $19 \mathrm{~h} 00$ min, enquanto que, no mesmo dia, às 19 h 01 min, foi capturado pelo anemômetro uma velocidade de $18.0 \mathrm{~km} / \mathrm{h}$ (Figura 9), equivalendo a $5.0 \mathrm{~m} / \mathrm{s}$ (Tabela 5).

Figura 8: Velocidade do vento na Estação Meteorológica de Observação de Superfície Automática

\begin{tabular}{|c|c|c|c|c|r|r|r|r|r|r|r|r|r|r|r|r|r|r|r|}
\hline Data & Hora & \multicolumn{1}{|c|}{ Temperatura $\left({ }^{\circ} \mathrm{C}\right)$} & \multicolumn{2}{|c|}{ Umidade $(\%)$} & \multicolumn{1}{|c|}{ Pto. Orvalho $\left({ }^{\circ} \mathrm{C}\right)$} & \multicolumn{3}{|c|}{ Pressão $(\mathrm{hPa})$} & \multicolumn{2}{|c|}{ Vento $(\mathrm{m} / \mathrm{s})$} & Radiação & Chuva \\
\hline & UTC & Inst. & Máx. & Mín. & Inst. & Máx. & Mín. & Inst. & Máx. & Mín. & Inst. & Máx. & Mín. & Vel. & Dir. $\left({ }^{\circ}\right)$ & Raj. & $\left(\mathrm{kJ} / \mathrm{m}^{2}\right)$ & $(\mathrm{mm})$ \\
\hline $30 / 09 / 2016$ & 18 & 32.0 & 32.8 & 31.1 & 31 & 33 & 30 & 12.7 & 13.5 & 12.3 & 957.9 & 958.7 & 957.8 & 4.8 & 57 & 9.8 & 2095. & 0.0 \\
\hline $30 / 09 / 2016$ & 19 & 31.1 & 32.9 & 31.1 & 33 & 33 & 30 & 12.9 & 13.2 & 12.4 & 957.8 & 957.9 & 957.7 & 5.1 & 72 & 8.2 & 1133. & 0.0 \\
\hline $30 / 09 / 2016$ & 20 & 30.1 & 31.3 & 30.1 & 37 & 37 & 32 & 13.8 & 13.8 & 12.5 & 958.0 & 958.0 & 957.8 & 5.1 & 86 & 9.4 & 500.0 & 0.0 \\
\hline
\end{tabular}

Fonte: Brasil (2016).

Figura 9: Velocidade capturado pelo anemômetro no dia 30 de setembro de 2016

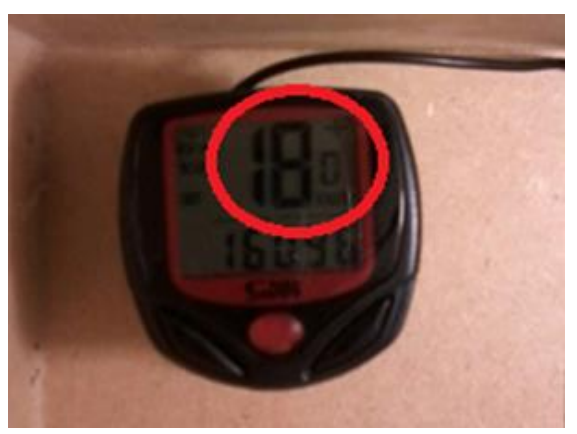

Fonte: Autoria própria.

Tabela 5: Medições de velocidade no dia 30 de setembro de 2016

\begin{tabular}{c|c|c|c}
\hline \multirow{2}{*}{ Número do dado } & \multirow{2}{*}{ Horário } & \multicolumn{2}{|c}{ Velocidade instantânea } \\
\cline { 3 - 4 } & & $\mathbf{k m} / \mathbf{h}$ & $\mathbf{m} / \mathbf{s}$ \\
\hline $\mathbf{5 2 3}$ & 18 h $51 \mathrm{~min}$ & 8.2 & 2.3 \\
\hline $\mathbf{5 2 4}$ & 18 h 56 min & 14.4 & 4.0 \\
\hline $\mathbf{5 2 5}$ & 19 h 01 min & 18.0 & 5.0 \\
\hline $\mathbf{5 2 6}$ & 19 h 06 min & 6.1 & 1.7 \\
\hline $\mathbf{5 2 7}$ & 19 h 11 min & 3.2 & 0.9 \\
\hline $\mathbf{5 2 8}$ & 19 h 16 min & 9.3 & 2.6 \\
\hline $\mathbf{5 2 9}$ & 19 h 21 min & 18.0 & 5.0 \\
\hline
\end{tabular}

Fonte: Autoria própria. 
Outra comparação demonstrada por meio das Figuras 10 e 11 é entre a Estação Meteorológica de Observação de Superfície Convencional, localizada na região central da cidade, com interferências de edificações e árvores, e o anemômetro instalado no UniDoctum, com o nível de interferência minimizada. A Figura 10 mostra que no dia 28 de outubro de 2016, às 18 h $00 \mathrm{~min}$, foi obtida uma velocidade de $2.6 \mathrm{~m} / \mathrm{s}$; e o anemômetro instalado na Instituição registrou uma velocidade de $15.4 \mathrm{~km} / \mathrm{h}$ (Figura 11), o que equivale a $4.3 \mathrm{~m} / \mathrm{s}$ (Tabela 6), dados distintos no mesmo horário, observando uma variação de velocidade, mesmo em menores alturas, em consonância com Almeida et al., 2017.

Figura 10: Velocidade do vento na Estação Meteorológica de Observação de Superfície Convencional

\begin{tabular}{|c|c|c|c|c|c|c|c|c|c|c|c|}
\hline \multirow[t]{2}{*}{ Data } & \multirow{2}{*}{\begin{tabular}{|l|} 
Hora \\
UTC
\end{tabular}} & \multirow{2}{*}{$\begin{array}{c}\text { Temperatura } \\
\left({ }^{\circ} \mathrm{C}\right)\end{array}$} & \multirow{2}{*}{$\begin{array}{c}\text { Umidade } \\
(\%)\end{array}$} & \multirow{2}{*}{\begin{tabular}{c|} 
Pressão \\
$(\mathrm{hPa})$
\end{tabular}} & \multicolumn{2}{|c|}{ Vento } & \multirow{2}{*}{$\begin{array}{c}\text { Nebulosidade } \\
\text { (Décimos) }\end{array}$} & \multirow{2}{*}{$\begin{array}{c}\text { Insolação } \\
(\mathrm{h})\end{array}$} & \multicolumn{3}{|c|}{ Dados diários } \\
\hline & & & & & $\begin{array}{l}\text { Vel. } \\
(\mathrm{m} / \mathrm{s})\end{array}$ & \begin{tabular}{|l|} 
Dir. \\
$\left({ }^{\circ}\right)$
\end{tabular} & & & $\begin{array}{l}\text { Temperatura } \\
\text { Máx. }\left({ }^{\circ} \mathrm{C}\right)\end{array}$ & $\begin{array}{l}\text { Temperatura } \\
\text { Mín. }\left({ }^{\circ} \mathrm{C}\right)\end{array}$ & $\begin{array}{l}\text { Chuva } \\
(\mathrm{mm})\end{array}$ \\
\hline $28 / 10 / 2016$ & 00 & 29.8 & 48 & 968.2 & 1.0 & 5 & 0 & 9.5 & 39.6 & & \\
\hline $28 / 10 / 2016$ & 12 & 31.6 & 45 & 969.6 & 1.5 & 5 & 0 & & & 23.2 & 0.0 \\
\hline $28 / 10 / 2016$ & 18 & 38.2 & 27 & 963.9 & 2.6 & 23 & 5 & & & & \\
\hline
\end{tabular}

Fonte: Brasil (2016).

Figura 11: Dados de 28/10/2016

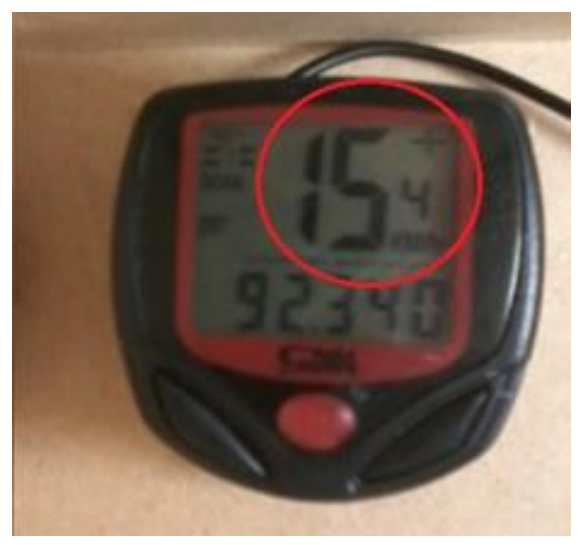

Fonte: Autoria própria.

Tabela 6: Medições de velocidade no dia 28 de outubro de 2016

\begin{tabular}{c|c|c|c}
\hline \multirow{2}{*}{ Número do dado } & \multirow{2}{*}{ Horário } & \multicolumn{2}{|c}{ Velocidade instantânea } \\
\cline { 3 - 4 } & & $\mathbf{k m} / \mathbf{h}$ & $\mathbf{~ m} / \mathbf{s}$ \\
\hline $\mathbf{4 2 6}$ & 17 h $56 \mathrm{~min}$ & 5.4 & 1.5 \\
\hline $\mathbf{4 2 7}$ & 18 h $00 \mathrm{~min}$ & 15.4 & 4.3 \\
\hline $\mathbf{4 2 8}$ & 18 h $06 \mathrm{~min}$ & 11.5 & 3.2 \\
\hline $\mathbf{4 2 9}$ & 18 h $11 \mathrm{~min}$ & 17.6 & 4.9 \\
\hline
\end{tabular}

Fonte: Autoria própria. 
Tendo como prática comum, nas análises do comportamento dos ventos com objetivos voltados ao aproveitamento energético, a utilização de modelos aproximados para descrever o perfil de velocidades (ALMEIDA et al., 2017) e diante dos resultados encontrados, validase que um dos aspectos principais para mensurar o potencial de viabilidade de geração de energia eólica é a velocidade do vento em $\mathrm{m} / \mathrm{s}$. Para que se possa garantir uma geração competitiva, é necessário no mínimo $5.0 \mathrm{~m} / \mathrm{s}$ no caso de geradores eólicos isolados. No entanto, para complexos eólicos, espera-se um vento de, ao menos, $7.0 \mathrm{~m} / \mathrm{s}$ (BOHME et al., 2016).

\subsection{Avaliação econômica da microgeração eólica}

Quando é considerado o uso de energia elétrica produzida por geração distribuída em uma rede de distribuição, há uma diminuição da tarifa de energia, devido à produção em grandes centrais geradoras, mesmo que seja uma redução mínima.

A partir dos dados apresentados na Tabela 7, desconsiderando-se a instalação dos equipamentos, pode-se verificar que a diminuição da tarifa de energia torna-se considerável quando há o aumento do número de usuários, tornando-se grande em aspecto de distribuição, e até mesmo para o consumidor, além de se estar investindo, produzindo e consumindo um bem próprio.

Tabela 7: Custo de equipamentos

\begin{tabular}{cc}
\hline Equipamento & Custo \\
\hline Aerogerador Istabreeze $-\mathbf{1 0 0 0} \mathbf{W}$, tensão de saída 24 V & 3849.00 \\
\hline Inversor On Grid Tie - 1000 W, tensão de saída 110 V & 1799.00 \\
\hline Total & 5648.00 \\
\hline
\end{tabular}

Fonte: Autoria própria.

Baseado no custo total de $\mathrm{R} \$ 5.648,00$ e considerando uma residência unifamiliar com consumo mensal de energia elétrica equivalente a $\mathrm{R} \$ 131,42$, daria para pagar este valor em 43 meses, ou seja, 3 anos e 7 meses. Isso pode ser ainda reduzido, passando necessariamente pela melhoria da competitividade nos custos de produção de equipamentos, uma vez que são influenciados fortemente pela aquisição dos insumos, pela escala de produção atingida, assim como pelo Custo Brasil (MELO, 2014). Nesse sentido, a redução nos preços dos 
equipamentos ainda é um dos maiores entraves para a diminuição do custo total de produção de energia eólica.

\section{CONSIDERAÇÕES FINAIS}

Atualmente a busca pela diminuição das tarifas de energia é constante, uma vez que as distribuidoras estão sobrecarregadas, o que faz com que a energia elétrica alcance o consumidor final com valores onerosos e aumenta a busca por gerações distribuídas.

A definição do potencial eólico do UniDoctum requereu coleta e análise sistemática de dados sobre a velocidade dos ventos, necessitando de uma avaliação rigorosa do levantamento de dados coletados no local e em estações meteorológicas de observação de superfície para fornecer uma estimativa do potencial de aproveitamento da energia eólica.

A construção da presente pesquisa possibilitou uma análise da viabilidade de instalação de um microaerogerador para que fosse utilizado como um complemento de geração de energia elétrica no UniDoctum, por meio de uma pesquisa de campo, com instalação de um anemômetro e métodos de distribuições estatísticas, para que fosse desenvolvido um indicador de incidência do vento do local.

A partir do anemômetro instalado no Bloco B da Instituição, fez-se o levantamento dos dados referentes a dois períodos de tempo para a análise da viabilidade. E esses atingiram valores superiores aos capturados pelas Estações Meteorológicas de Observação de Superfície do Município no mesmo espaço de tempo, sendo de grande importância para o indicador de viabilidade.

Apesar da pouca atuação dos ventos, os resultados apontam para viabilidade da implantação da microgeração, mesmo com uma média abaixo do esperado e com grande variabilidade dos dados, modelados à distribuição de Weibull, ferramenta essencial para auxílio do controle e previsão da velocidade dos ventos, em que atuou de forma robusta na consequente tomada de decisão na área de estudo desta pesquisa. Mediante esse fato, verificou-se que é necessário um estudo com o anemômetro instalado a uma altura superior a 13 m para garantir um melhor aproveitamento da captação da velocidade mínima do vento, na implementação da microgeração eólica, e para que se possa ter mais confiabilidade na quantidade de energia gerada, visto que quanto mais alto o aerogerador, maior será a quantidade de energia eólica gerada e a potência instalada. 


\section{REFERÊNCIAS}

AGÊNCIA NACIONAL DE ENERGIA ELÉTRICA. Resolução normativa n. ${ }^{\circ}$ 687, de 24 de novembro de 2015. Altera a Resolução normativa n. ${ }^{\circ}$ 482, de 17 de abril de 2012, e os Módulos 1 e 3 dos Procedimentos de Distribuição - PRODIST. Disponível em: https://www2.aneel.gov.br/cedoc/ren2015687.pdf. Acesso em: 9 abr. 2020.

ALLAN, R. N.; BILLINTON, R. Reliability evaluation of engineering systems: concepts and techniques. 2. ed. New York: Plenum Press, 1992.

ALMEIDA, M. A. M. et al. Análise da camada limite atmosférica no complexo eólico Amontada. In: CONGRESSO DE INOVAÇÃO TECNOLÓGICA EM ENERGIA ELÉTRICA, 9., 2017, João Pessoa. Anais [...]. Brasil: ANEEL, 2017. Disponível em: http://www.citenel.gov.br/pages/exporta_trabalhos.aspx. Acesso em: 9 abr. 2020.

BOHME, G. S. et al. Análise das etapas de desenvolvimento de projetos de energia eólica - Estudo de caso. In: ENCONTRO INTERNACIONAL SOBRE GESTÃO EMPRESARIAL E MEIO AMBIENTE, 18., 2016, São Paulo. Anais [...]. Brasil: USP, 2016. Disponível em: http://engemausp.submissao.com.br/18/anais/arquivos/456.pdf. Acesso em: 9 abr. 2020.

BRAGA, N. C. Projetos eletrônicos educacionais com energia alternativa. São Paulo: NCB, 2013.

BRASIL. Ministério da Agricultura, Pecuária e Abastecimento. Instituto Nacional de Meteorologia. Brasília, Distrito Federal. Teófilo Otoni: Estação Meteorológica de Observação de Superfície, 2016.

BRASIL. Ministério das Minas e Energia. Empresa de Pesquisa Energética. Anuário Estatístico de Energia Elétrica 2019. Rio de Janeiro: Ministério das Minas e Energia, dez. 2019. Disponível em: http://www.epe.gov.br/pt/publicacoes-dadosabertos/publicacoes/anuario-estatistico-de-energia-eletrica. Acesso em: 9 abr. 2020.

BRASIL. Ministério das Minas e Energia. Empresa de Pesquisa Energética. Plano Decenal de Expansão de Energia 2029. Rio de Janeiro: Ministério das Minas e Energia, 2020. Disponível em: http://www.epe.gov.br/sites-pt/publicacoes-dadosabertos/publicacoes/Documents/PDE\%202029.pdf. Acesso em: 13 abr. 2020.

COMPANHIA ENERGÉTICA DE MINAS GERAIS S.A. Manual de distribuição. ND5.30. Requisitos para a conexão de acessantes ao sistema de distribuição Cemig D - Conexão em baixa tensão. Belo Horizonte: CEMIG, 2019. Disponível em: http://www.cemig.com.br/pt-

br/atendimento/Clientes/Documents/Normas\%20T\%C3\%A9cnicas/ND.5.30.pdf. Acesso em: 9 abr. 2020.

GOOGLE MAPS, 2020. Disponível em: https://www.google.com.br/maps/@-17.8533722,41.4842773,304m/data=!3m1!1e3. Acesso em: nov. 2016. 
JONG, P.; KIPERSTOK, A.; TORRES, E. A. Economic and environmental analysis of electricity generation technologies in Brazil. Renewable and Sustainable Energy Reviews, v. 52, p. 725-739, 2015. Disponível em: https://doi.org/10.1016/j.rser.2015.06.064. Acesso em: 13 abr. 2020.

MELO, E. Investimentos em energia eólica no Brasil: aspectos de inserção, tecnologia e competitividade. Concreto \& Construções, São Paulo, ed. 75, p. 74-77, jul-set 2014. Disponível em: http://ibracon.org.br/publicacoes/revistas_ibracon/rev_construcao/pdf/Revista_Concreto_7 5.pdf. Acesso em: 13 abr. 2020.

PAZHERI, F. R.; OTHMAN, M. F.; MALIK, N. H. A review on global renewable electricity scenario. Renewable and Sustainable Energy Reviews, v. 31, p. 835-845, 2014. Disponível em: https://doi.org/10.1016/j.rser.2013.12.020. Acesso em: 13 abr. 2020.

PESTANA, D. D.; VELOSA, S. F. Introdução à probabilidade e à estatística. 4. ed. Lisboa: Fundação Calouste Gulbenkian, 2008. 1164 p.

R PROJECT. The R Project for statistical computing. Versão 3.2.4: R Project, 2014. Disponível em: https://www.r-project.org/. Acesso em: nov. 2016.

SCHMIDT, J.; CANCELLA, R.; PEREIRA JÚNIOR, A. O. An optimal mix of Schmidt PV, wind and hydro power for a low-carbon electricity supply in Brazil. Renewable Energy, v. 85, p. 137-147, 2016. Disponível em: https://doi.org/10.1016/j.renene.2015.06.010. Acesso em: 13 abr. 2020.

TRIOLA, M. F. Introdução à estatística. 10. ed. Rio de Janeiro: LTC. 2008. 